\title{
Caracterização clínica e molecular de 211 pacientes brasileiros com a doença de Machado-Joseph
}

\author{
Clinical and molecular characterization of 211 Brazilian patients with Machado-Joseph \\ disease
}
Caracterización clínica y molecular de 211 pacientes brasileños con la enfermedad de Machado-Joseph

Giovanna Cordeiro Ferreira ${ }^{1}$, Murilo Junqueira Cavalari Dias ${ }^{1}$, Pedro José Tomaselli², Sandra Elisabete Marques², Vívian Pedigone Cintra ${ }^{1,2 *}$, Wilson Marques Júnior².

\section{RESUMO}

Objetivo: Avaliar as características clínicas e genéticas de um grande grupo de acometidos pela ataxia espinocerebelar tipo 3 (AEC3), conhecida por Doença de Machado-Joseph (DMJ). Métodos: 211 pacientes AEC3/DMJ foram caracterizados de acordo com o sexo, idade de início da doença, número de tripletos CAG (Citosina-Adenina-Guanina) no alelo expandido, subtipo, sexo do progenitor do qual herdou a doença, antecipação dos sinais e sintomas e percentual da prole afetada. Resultados: Foi observado que as maiores expansões CAG, o subtipo 1 da doença e o sexo do paciente influenciam na idade de início da patologia. Indivíduos do sexo masculino desenvolveram sinais e sintomas de AEC3/DMJ quatro anos antes do que indivíduos do sexo feminino. A média da idade de início dos pacientes foi de 4,84 anos anterior à idade de início dos genitores, indicando antecipação. Ademais, foi observado que a antecipação paterna foi de 7,19 anos, enquanto a materna foi de 3,57 anos. Conclusão: Esta população de AEC3/DMJ mostra algumas peculiaridades, principalmente em relação às diferentes idades de início entre homens e mulheres. $O$ reconhecimento de fatores que modificam a idade de início da doença e a maneira como eles interferem na população afetada podem contribuir para o futuro manejo dessa condição.

Palavras-chave: Doença de Machado-Joseph, Ataxia espinocerebelar, Expansão das repetições de trinucleotídeos, Antecipação genética, Idade de início.

\begin{abstract}
Objective: To evaluate the clinical and genetic characteristics of a large group of people affected by spinocerebellar ataxia type 3 (SCA3), also known as Machado-Joseph disease (MJD). Methods: 211 SCA3/MJD patients were characterized according to sex, age of disease onset, number of CAG repeats in the expanded allele, subtype, sex of the parent from whom the disease was inherited, anticipation of signs and symptoms and percentage of affected offspring. Results: We observed that larger CAG expansions, subtype 1 of the disorder and the patient's gender influence the earlier onset of the disease. Male developed the signs and symptoms of SCA3/MJD four years earlier than female. The mean age at onset in patients was 4.84 years earlier than the mean age at onset of their parents, indicating anticipation. In addition, it was observed that the paternal anticipation was 7.19 years while the maternal was 3.57 years. Conclusion: This SCA3/MJD population shows some peculiarities, especially in relation to the different ages of onset between male and female. The recognition of factors that modify the age of onset of the disease and the way they interfere in the affected population can contribute to the future management of this condition.
\end{abstract}

Keywords: Machado-Joseph disease, Spinocerebellar ataxia, Trinucleotide repeat expansion, Genetic anticipation, Age of onset.

\footnotetext{
${ }^{1}$ Centro Universitário Municipal de Franca (Uni-FACEF), Franca - SP.

*E-mail: vivianpedigone@yahoo.com.br

2 Universidade de São Paulo (USP), Ribeirão Preto - SP.
} 


\section{RESUMEN}

Objetivo: Evaluar las características clínicas y genéticas de un gran grupo de personas afectadas por ataxia espinocerebelosa tipo 3 (AEC3), también conocida como enfermedad de Machado-Joseph (MJ). Métodos: 211 pacientes con $A E C 3 / M J$ fueron caracterizados según el sexo, edad de inicio de la enfermedad, número de tripletes CAG en el alelo expandido, subtipo, sexo del progenitor del que se heredó la enfermedad, anticipación de signos y síntomas y el porcentaje de descendencia afectada. Resultados: Se identificó que las grandes expansiones de CAG, el subtipo 1 de la enfermedad y el género del paciente influyen en la edad de inicio del transtorno. Los hombres desarrollaron signos y síntomas de AEC3/MJ cuatro años antes que las mujeres. La edad media de inicio de los pacientes fue de 4,84 años antes de la edad de inicio de sus padres, lo que indica anticipación. Además, se observó que la anticipación paterna fue de 7,19 años, mientras que la anticipación materna fue de 3,57 años. Conclusión: Esta población de AEC3/MJ muestra algunas peculiaridades, principalmente en relación con las diferentes edades de inicio entre hombres y mujeres. EI reconocimiento de los factores que modifican la edad de aparición de la enfermedad y la forma en que interfieren en la población afectada pueden contribuir al futuro manejo de esta patología.

Palabras clave: Enfermedad de Machado-Joseph, Ataxia espinocerebelosa, Expansión de repetición de trinucleótido, Anticipación genética, Edad de inicio.

\section{INTRODUÇÃO}

As ataxias espinocerebelares (AEC) são doenças autossômicas dominantes caracterizadas por lenta e progressiva incoordenação generalizada da marcha, fala e movimentos, por consequência do acometimento cerebelar e de suas principais conexões (BETTENCOURT C e LIMA M, 2011). Podem ainda apresentar outras manifestações neurológicas e não neurológicas, como incoordenação apendicular, disartria, neuropatia periférica, disfagia, nistagmo, retinopatia e tremor de intenção, em decorrência do acometimento de outras estruturas como núcleos da base e do tronco encefálico, trato corticoespinal, coluna posterior da medula espinal e retina. (BETTENCOURT C e LIMA M, 2011; ZHANG B, et al., 2012).

Até o momento, pelo menos 44 loci e 34 genes relacionados à AEC já foram mapeados e 48 subtipos de ataxias espinocerebelares foram descritas. O tipo de mutação mais frequente é a expansão do trinucleotídeo CAG (Citosina-Adenina-Guanina) responsável pelas AEC1, AEC2, AEC3, AEC6, AEC7, AEC17 e ADRPL (Atrofia Dentatorrubro Palidoluisiana) (WYNER N, et al., 2020).

Entre todas as AEC, a mais comum é a AEC3, também conhecida como Doença de Machado Joseph (DMJ), presente em diversos grupos étnicos. A frequência relativa da AEC3/DMJ é alta no Brasil (70 - 92\%) e, na região nordeste do estado de São Paulo, a prevalência de 5 acometidos a cada 100.000 habitantes é uma das mais altas do país (TEIVE HG, et al., 2019; CINTRA VP, et al., 2014; DE CASTILHOS RM, et al., 2014). A mutação causadora na $A E C 3 / D M J$ consiste na expansão de um trato $C A G$ instável no éxon 10 do gene ATXN3 (BETTENCOURT C e LIMA M, 2011). Consensualmente, os alelos normais para AEC3/DMJ variam de 11 a 44 trinucleotídeos CAG e as expansões do gene ATXN3 apresentam de 60 a 87 repetições, embora já tenham sido relatados indivíduos sintomáticos contendo de 45 a 59 tripletos (SEQUEIROS J, et al., 2010).

Sabe-se que o produto genético oriundo da expansão CAG produz um traço poliglutamínico, mas o mecanismo pelo qual o número aumentado de repetições de poliglutamina danifica os neurônios não está claro. A AEC3/DMJ é caracterizada por apresentar um alto grau de heterogeneidade clínica, bem como uma grande diversificação no grau de incapacidade resultante (CHEN YS, et al., 2020; FURTADO GV, et al., 2019). Até o momento, oito subtipos clínicos foram associados à doença, podendo ser identificados, além dos sinais cerebelares, distonia, sinais piramidais, oftalmoparesia, neuropatia periférica, tríade parkinsoniana, paraparesia espástica e doença do neurônio motor (FARRAR MA, et al., 2016; MORO A, et al., 2014)

A idade de início dos sinais e sintomas da AEC3/DMJ ocorre geralmente entre 34 e 40 anos, embora extremos de cinco e 70 anos tenham sido relatados (PAULSON H, 2012). A expansão do gene ATXN3 é apontada como o principal modificador da idade de início, entretanto explica apenas parcialmente (entre 50 a $75 \%$ ) a variabilidade da idade de início da AEC3/DMJ (AKÇIMEN F, et al., 2020). Ademais, a idade de início também pode ser influenciada pelo fenômeno de antecipação, o qual consiste em um aumento das repetições CAG nos alelos expandidos dos descendentes (BETTENCOURT C e LIMA M, 2011). 
Assim enunciado, sabe-se que as AEC são doenças neurodegenerativas que causam grande impacto, tanto para o médico, quanto para o paciente e seus familiares. Como já elencado, a AEC3/DMJ, por ser a mais predominante no Brasil (70\%-92\%), foi escolhida como objeto de estudo, uma vez que o reconhecimento e a identificação dos fatores modificadores da doença e a forma como interferem na população acometida podem contribuir para o futuro manejo dessa condição.

O presente estudo tem por objetivo avaliar as características clínicas, moleculares e demográficas de um grande grupo de pacientes com AEC3/DMJ diagnosticados com a mutação na região sudeste do Brasil, principalmente na mesorregião de Ribeirão Preto.

\section{MÉTODOS}

\section{Participantes da pesquisa}

Ao total, foram incluídos 211 pacientes geneticamente identificados com a expansão CAG no lócus causador da AEC3/DMJ. Todos os pacientes foram clinicamente avaliados em ambulatório de Neurogenética em uma cidade de São Paulo e os exames moleculares para diagnóstico confirmatório foram realizados de acordo com o protocolo previamente estabelecido (SEIDEL K, et al., 2012). O presente trabalho foi autorizado pelo Comitê de Ética em Pesquisa Médica, relativo ao processo HCRP № 11026/2008.

\section{Classificação das variáveis demográficas}

A idade de início da doença foi caracterizada como o período em que foram percebidas as primeiras alterações sintomatológicas, sendo majoritariamente relatadas a instabilidade na marcha, seguida ou não por quedas. Embora minoritariamente, diplopia e fala mal articulada também foram relatadas no início da doença. Ademais, a antecipação dos sinais e sintomas foi estabelecida como a diferença entre a idade de início apresentada pelo genitor e a idade de início do paciente em estudo.

Para o cálculo do percentual da prole afetada em cada família, foi considerada para cada paciente, a quantidade total de irmãos sadios e afetados. Como sadios, foram incluídos os irmãos com ausência da expansão CAG detectada por exame molecular e, como afetados, foram incluídos os irmãos com doença claramente manifesta ou os indivíduos assintomáticos com teste molecular preditivo positivo para a expansão CAG. Foram excluídos os filhos únicos, cujos percentuais possíveis (0 ou 100\%) poderiam resultar em viés de amostragem.

\section{Classificação dos subtipos clínicos}

A classificação dos pacientes em cada subtipo da doença envolveu a análise de um conjunto de sinais obtidos no exame neurológico completo. Foram excluídos os indivíduos que apresentaram tempo de evolução da doença inferior a cinco anos. Para fins de classificação, os dados clínicos de todos os pacientes foram interpretados pelo mesmo neurologista (Quadro 1).

Quadro 1 - Classificação e apresentação clínica.

\begin{tabular}{|c|l|}
\hline Classificação & \multicolumn{1}{c|}{ Sinais Clínicos } \\
\hline Subtipo 1 & $\begin{array}{l}\text { Ataxia de marcha e apendicular, oftalmoparesia externa progressiva (OEP), distonia e } \\
\text { sinais piramidais marcados. }\end{array}$ \\
\hline Subtipo 2 & Déficits predominantemente cerebelares e piramidais com OEP e ausência de distonia. \\
\hline Subtipo 3 & $\begin{array}{l}\text { Ataxia cerebelar com a presença variável de OEP e sinais piramidais, mas com neuropatia } \\
\text { sensitiva e/ou motora. }\end{array}$ \\
\hline Subtipo 4 & $\begin{array}{l}\text { Tríade parkinsoniana (rigidez, bradicinesia e tremor de repouso) associada a sinais } \\
\text { cerebelares leves e presença variável de neuropatia sensitivo-motora ou amiotrofia }\end{array}$ \\
\hline Subtipo 5 & Paraparesia espástica mais marcante que a ataxia de marcha \\
\hline Subtipo 6 & Ataxia cerebelar pura \\
\hline Subtipo 7 & $\begin{array}{l}\text { Forma mista de ataxia e parkinsonismo responsivo à levodopa. Ataxia muito leve, sinais } \\
\text { piramidais marcados, com espasticidade importante e parkinsonismo predominantemente } \\
\text { rígido-acinético, estando ausentes tremor, neuropatia sensitivo-motora e amiotrofia. }\end{array}$ \\
\hline $\begin{array}{c}\text { Doença do } \\
\text { Neurônio } \\
\text { Motor (DMN) }\end{array}$ & $\begin{array}{l}\text { Sinais cerebelares e piramidais variáveis, com desnervação nos segmentos craniano, } \\
\text { cervical, torácico e lombossacro evidenciados pela eletromiografia, confirmando a doença } \\
\text { do neurônio motor inferior }\end{array}$ \\
\hline
\end{tabular}

Fonte: FERREIRA GC, et al., 2020. 


\section{Análise estatística}

Toda a análise estatística foi realizada utilizando o software SPSS Statistics v.21. Para os cálculos da idade de início e antecipação foi utilizado o teste-t e, para o percentual da prole afetada, o teste não paramétrico de Mann Whitney. ANOVA com correção de Bonferroni e teste do qui-quadrado foram empregados nos cálculos envolvendo o subtipo da doença. Para os testes envolvendo duas variáveis numéricas, como a idade de início dos sintomas e número de trinuceotídeos $C A G$, foi utilizado o teste de correlação de Pearson.

\section{RESULTADOS}

\section{Idade de início dos sinais e sintomas da doença}

O estudo do tamanho das expansões dos tripletos revelou correlação significativa negativa $(r=-0,73)$ entre o comprimento da repetição CAG e a idade de início da doença $\left(p<1 \times 10^{-6}\right)$, mostrando que, quanto mais precoce é a idade de início da AEC3/DMJ, maior é a expansão CAG (Tabela 1).

Tabela 1 - Coeficiente de correlação de Pearson entre a média da idade de início e o número de tripletos CAG no alelo expandido para cada subtipo clínico.

\begin{tabular}{|c|c|c|c|c|c|c|}
\hline \multirow{2}{*}{$\begin{array}{l}\text { Subtipo } \\
\text { clínico }\end{array}$} & \multirow{2}{*}{$\begin{array}{l}\bar{X} \pm \text { DP da idade } \\
\text { de início (anos) }\end{array}$} & \multicolumn{3}{|c|}{ № de tripletos CAG no alelo expandido } & \multirow{2}{*}{$\begin{array}{c}\text { Correlação de } \\
\text { Pearson (r) }\end{array}$} & \multirow{2}{*}{ p-valor } \\
\hline & & $\bar{X} \pm \mathrm{DP}$ & Min-Max & Mediana & & \\
\hline Geral & $38,65 \pm 12,27$ & $70,82 \pm 4,33$ & $58-80$ & 71 & $-0,765$ & $<1 \times 10^{-6}$ \\
\hline 1 & $26,65 \pm 7,58$ & $75,35 \pm 3,01$ & $69-80$ & 76 & $-0,665$ & $1 \times 10^{-3}$ \\
\hline 2 & $35,56 \pm 9,81$ & $71,60 \pm 3,92$ & $58-79$ & 72 & $-0,659$ & $2 \times 10^{-6}$ \\
\hline 3 & $46,82 \pm 8,63$ & $69,00 \pm 3,22$ & $63-75$ & 69,50 & $-0,625$ & $2 \times 10^{-3}$ \\
\hline 6 & $43,74 \pm 11,79$ & $68,42 \pm 3,46$ & $62-75$ & 69 & $-0,600$ & $3,6 \times 10^{-4}$ \\
\hline DNM & $41,14 \pm 14,93$ & $70,07 \pm 5,03$ & $63-80$ & 69 & $-0,887$ & $2,3 \times 10^{-5}$ \\
\hline
\end{tabular}

Legenda: Média; DP: desvio padrão. Fonte: FERREIRA GC, et al., 2020.

Utilizando-se o número CAG do alelo expandido como covariável, as médias das idades de início foram de 36,12 anos para o sexo masculino e de 40,29 anos para o feminino, havendo diferença significativa entre elas $(p=0,028)$. Tal diferença é evidenciada na Figura 1, uma vez que $64,6 \%$ dos homens da presente população desenvolveram sinais e sintomas da doença anterior aos 40 anos de idade, enquanto $55,1 \%$ das mulheres iniciaram os primeiros sinais após essa idade.

Figura 1 - Distribuição das diferentes frequências de idade de início da AEC/DMJ em cada sexo.

Sexo

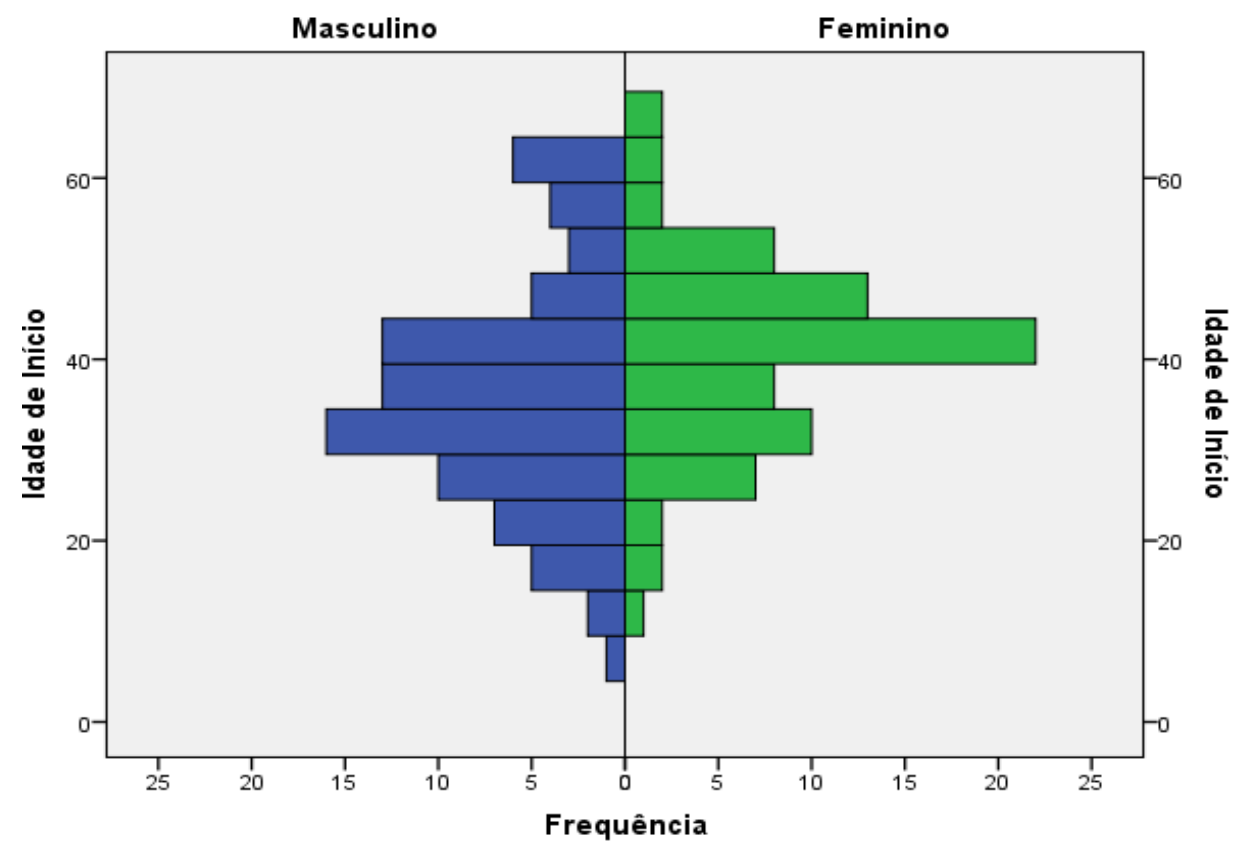

Fonte: FERREIRA GC, et al., 2020. 
Os subtipos clínicos da $A E C 3 / D M J$ identificados no presente trabalho, por ordem decrescente de frequência, foram os subtipos $2(34,8 \%), 6(23,9 \%), 3(14,9 \%), 1(12,9 \%)$, DNM $(9 \%), 4(2,6 \%), 7(1,3 \%)$ e 5 $(0,6 \%)$. Devido ao reduzido número de pacientes identificados, foram excluídos das análises estatísticas, os grupos 4, 5 e 7. Correlacionou-se também a idade de início de cada subtipo clínico e o número de tripletos CAG do alelo expandido, obtendo-se correlação negativa e significância em todos os subtipos (Tabela 1).

Embora não tenha existido predomínio de qualquer sexo entre os pacientes dos subtipos clínicos $1(\mathrm{p}=$ $0,37), 2(p=0,17), 3(p=0,39)$ e $6(p=0,86)$, encontrou-se diferença significativa $(p=0,033)$ entre os percentuais do sexo masculino $(78,6 \%)$ e feminino $(21,4 \%)$ no subtipo DNM.

Ademais, houve diferença estatística significativa $\left(p<1 \times 10^{-6}\right)$ entre as médias da idade de início de acordo com os subtipos clínicos. Os indivíduos do subtipo 1 apresentaram a média da idade de início mais precoce da doença, aos 26,65 anos, seguido pelos subtipos 2 (35,56 anos), DNM (41,14 anos) e 6 (43,74 anos) (Tabela 1).

Os pacientes classificados no subtipo 3 apresentaram a média de idade de início mais tardia, aos 46,82 anos (Tabela 1). Comparando-se as médias entre os subtipos, encontrou-se diferença significativa entre os subtipos 1 e $2(p=0,021) ; 1$ e $3\left(p<1 \times 10^{-6}\right) ; 1$ e $6\left(p=1 \times 10^{-6}\right) ; 1$ e DNM $(p=0,001) ; 2$ e $3(p=0,001) ; 2$ e 6 $(p=0,012)$.

Mesmo utilizando o número de tripletos CAG do alelo expandido como covariável, a diferença das médias de idade de início para cada subtipo clínico permaneceu significativa $(p=0,026)$. A Figura 2 evidencia tais diferenças, à medida que demonstra a frequência das idades de início para cada subtipo clínico.

Figura 2 - Distribuição de diferentes frequências de idade de início da AEC/DMJ em cada subtipo clínico.

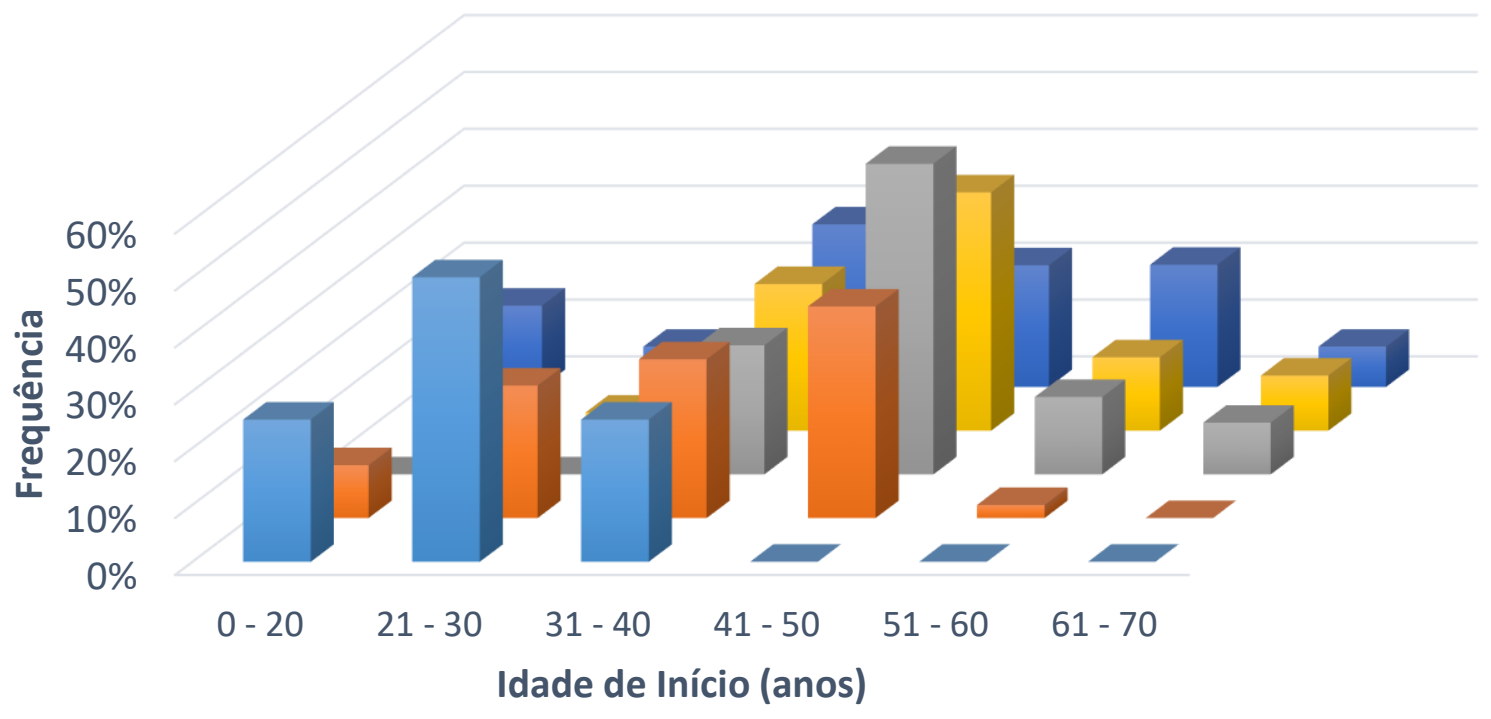

Subtipo 1 Subtipo 2 Subtipo $3 \square$ Subtipo $6 \square$ DNM

Fonte: FERREIRA GC, et al., 2020.

Ademais, foi observado que as três variáveis, número de trinucleotídeos CAG do alelo expandido $(\mathrm{p}<1 \mathrm{x}$ $\left.10^{-6}\right)$, sexo do paciente $(p=0,05)$ e subtipo clínico $(p=0,01)$, também influenciam em conjunto, a idade de início dos sinais e sintomas da AEC/DMJ em cada paciente.

Não houve diferença significativa entre as médias da idade de início do paciente de acordo com o sexo do genitor $(p=0,71)$ e também não existiu predomínio do sexo do genitor em cada subtipo da doença $(p=0,10)$. A Tabela 2 apresenta as médias de idade de início de acordo com o gênero do paciente, o sexo do genitor e o subtipo clínico, bem como o seu nível de significância. 
Tabela 2 - Médias de idade de início da AEC/DMJ de acordo com o gênero do paciente, o sexo do genitor e o subtipo clínico.

\begin{tabular}{lccc} 
Características & \multicolumn{2}{c}{ Média da idade de início \pm DP } & \multirow{2}{*}{ p-valor } \\
\cline { 2 - 3 } Clínicas & $36,12 \pm 12,82$ & Feminino & \\
\hline Sexo do paciente & $37,41 \pm 11,40$ & $40,29 \pm 10,66$ & $0,028^{*}$ \\
Sexo do genitor & $25,33 \pm 7,38$ & $38,12 \pm 11,74$ & 0,708 \\
Subtipo 1 & $31,12 \pm 9,46$ & $28,63 \pm 7,93$ & 0,371 \\
Subtipo 2 & $44,54 \pm 7,76$ & $38,46 \pm 9,07$ & 0,170 \\
Subtipo 3 & $43,13 \pm 16,03$ & $50,11 \pm 9,18$ & 0,394 \\
Subtipo 6 & $40,36 \pm 16,76$ & $44,31 \pm 6,13$ & 0,857 \\
Subtipo DNM & $44,00 \pm 5,29$ & $0,033^{*}$
\end{tabular}

Legenda: DP: desvio-padrão; * Diferença estatisticamente significativa $(p \leq 0,05)$

Fonte: FERREIRA GC, et al., 2020.

\section{Antecipação}

A média da idade de início da AEC3/DMJ dos pacientes na presente amostra (34,9 anos) foi significativamente inferior $\left(p=8 \times 10^{-6}\right)$ à média da idade de início dos seus genitores $(39,73$ anos), representando uma antecipação de sinais e sintomas da doença de 4,84 anos (Tabela 3). Ademais, foi observado que a média de antecipação de origem paterna foi de 7,19 anos enquanto a de origem materna foi de 3,57 anos, indicando forte tendência à diferença significativa entre elas $(p=0,056)$ (Tabela 3).

A antecipação significativa da doença foi também encontrada no subtipo clínico 1 (média de idade de início do paciente aos 25 anos e média de idade de início do genitor aos 35,08 anos) e subtipo 6 (média da idade de início do paciente aos 37,86 anos e média de idade de início do genitor aos 43,21 anos).

Embora a antecipação em pacientes do sexo masculino $\left(-6,24, p=3 \times 10^{-6}\right)$ seja superior à antecipação em pacientes do sexo feminino $(-3,26, p=0,049)$ não houve diferença significativa entre ambos os sexos $(p=$ $0,15)$ (Tabela 3).

Tabela 3 - Antecipação dos sinais e sintomas da AEC/DMJ de acordo com o gênero do paciente, o sexo do genitor e o subtipo clínico.

\begin{tabular}{lcc}
\hline $\begin{array}{l}\text { Características } \\
\text { Clínicas }\end{array}$ & $\begin{array}{c}\text { Antecipação dos sinais e } \\
\text { sintomas (anos) }\end{array}$ & p-valor \\
\hline Geral & $-4,84 \pm 8,96$ & $1,1 \times 10^{-5 *}$ \\
\hline Transmissão paterna & $-7,19 \pm 6,58$ & $0,056^{\star *}$ \\
Transmissão materna & $-3,57 \pm 9,99$ & 0,15 \\
\hline Paciente do sexo $\hat{\lambda}$ & $-6,24 \pm 7,35$ & $2,6 \times 10^{-5 *}$ \\
Paciente do sexo + & $-3,26 \pm 10,53$ & 0,06 \\
\hline Subtipo 1 & $-10,08 \pm 5,51$ & 0,53 \\
\hline Subtipo 2 & $-4,00 \pm 8,71$ & $0,05^{*}$ \\
\hline Subtipo 3 & $-1,83 \pm 9,71$ & 0,136 \\
\hline Subtipo 6 & $-5,35 \pm 9,47$ & \\
\hline Subtipo DNM & $-6,001$ & \\
\hline
\end{tabular}

Legenda: * Diferença estatisticamente significativa $(\mathrm{p} \leq 0,05)$; ${ }^{*}$ Fortíssima tendência da transmissão paterna ter mais antecipação que a transmissão materna; ${ }^{1}$ Diferença entre as medianas da idade de início do genitor $(47,00)$ e do paciente $(41,00)$.

Fonte: FERREIRA GC, et al., 2020.

\section{Percentual da prole afetada}

Na presente amostra, os genitores do sexo masculino transmitiram o alelo expandido para $42,1 \%$ de sua prole, enquanto os genitores do sexo feminino o transmitiram $41,1 \%$ das vezes. Não houve diferença significativa entre os sexos dos genitores para o cálculo da prole afetada $(p=0,86)$. 


\section{DISCUSSÃO}

O elevado grau de variabilidade na idade de início da AEC3/DMJ está inversamente relacionada com o tamanho da expansão da região (CAG)n no gene ATXN3 (BETTENCOURT C e LIMA M, 2011). Consoante com a literatura, o presente estudo revelou uma correlação negativa entre o alelo expandido e a idade de início, demonstrando que quanto maior a repetição CAG, menor a idade de apresentação dos primeiros sintomas (BETTENCOURT C e LIMA M, 2011; DONIS K C, et al., 2016).

A amostra estudada apresentou idades de início variando dos sete aos 66 anos, com o número de repetições de 58 a 80 trinucleotídeos. Os maiores números de repetições $(78,79$ e 80$)$ foram encontrados em heterozigose entre os pacientes com idades de início mais precoces (entre os 13 e 21 anos).

No presente estudo, a média da idade de início da AEC3/DMJ foi de 38,13 anos, três anos mais tardia do que na região sul do Brasil, com 35, e similar à idade de início portuguesa, de 37,83 anos ( MERGENER R, et al., 2020; DE MATTOS EP, et al., 2019; VALE J, et al., 2010). Sabe-se que quanto maior o parentesco, mais homogênea a idade de início, portanto levanta-se a hipótese de que a genética da população aqui estudada seja distinta da população sul rio-grandense e similar à população portuguesa, justificando, em partes, diferentes médias de idade em um mesmo país (MARTINS S, et al, 2014).

Nosso estudo mostrou que o início da doença ocorre 4,17 anos mais precocemente no sexo masculino. Poucos estudos reportaram diferença nas médias das idades entre os gêneros. Chen $\mathrm{S}$, et al. (2015) demonstraram que o polimorfismo mitocondrial 10398A antecipa em três anos a idade de início no sexo masculino. Bettencourt C, et al. (2011) apresentaram uma modificação existente na APOE2, a qual aumenta o risco para o desenvolvimento precoce da doença no sexo masculino em cinco anos. Mais estudos são necessários para determinar se essas alterações estão presentes na população aqui estudada.

Paralelamente, outros genes como ATN1, ATXN1, ATXN2, ATXN7, CACNA1A, C9orf72, KCNN3, HTT e $R A / 1$ já foram reportados como exercendo alguma influência na idade de início da AEC3/DMJ (CHEN Z, et al., 2016; DU MONTCEL S, et al., 2014; WANG C, et al., 2015)

A classificação da AEC3/DMJ em subtipos clínicos é baseada em fatores que nos permitem diferenciá-los quanto a progressão, o desenvolvimento de sinais e sintomas e até mesmo a idade de início da doença. Na série apresentada neste estudo, o subtipo clínico mais comumente encontrado foi o tipo 2, que é a forma clássica descrita na literatura (MORO A, et al., 2014). É possível que, durante o curso da doença, novos sintomas possam surgir e alterar o fenótipo do paciente.

No presente trabalho, esse viés foi reduzido, uma vez que os pacientes que apresentaram tempo de evolução da doença inferior a cinco anos foram excluídos de todas as análises estatísticas que envolvessem o subtipo clínico. Embora em percentual reduzido, os subtipos 4, 5 e 7 também foram encontrados em nossa série, sugerindo que os fenótipos de parkinsonismo, paraparesia espástica e ataxia mista não são raros e devem ser incluídos no rol de apresentação clínica da AEC3/DMJ, reduzindo assim, o número de subdiagnósticos.

Apesar de o presente estudo não ter utilizado a média da idade de início dos sinais e sintomas da doença como fator de critério de classificação dos pacientes em diferentes subtipos clínicos, as diferenças encontradas entre tais médias obedeceram ao padrão descrito na literatura. É sabido que os três primeiros subtipos da doença apresentam parâmetros característicos de idade de início, nos quais, o subtipo 1 apresenta idade de início mais precoce, enquanto o 3 tem a idade inicial mais tardia (BETTENCOURT $\mathrm{C}$ e LIMA M, 2011).

Ao comparar as médias de idade de início entre os subtipos clínicos, foi encontrada uma diferença significativa entre o subtipo 1 e os demais subtipos (2,3,6 e DNM), já relatada por vários autores, como Teive H G, et al. (2019) e Moro A, et al. (2014). Ademais, houve significativa diferença entre as médias do subtipo 2 (35,56 anos) e 6 (43,74 anos). Os subtipos 2 e 3 também apresentaram diferentes médias de idade de início, que também foram relatadas por Bettencourt C e Lima M (2011) e Moro A, et al. (2014). Mesmo utilizando o número de tripletos CAG do alelo expandido como covariável, a diferença das médias de idade de início para cada subtipo clínico permaneceu significativa. 
Visto que o subtipo 1 da doença apresenta maior precocidade dos sinais e sintomas e que o predomínio do sexo masculino neste grupo poderia parcialmente justificar o início mais precoce da doença em homens na presente população, também se avaliou a relação entre os subtipos clínicos e o sexo do paciente. Entretanto, nenhuma associação pôde ser observada, assim como nenhuma relação foi identificada nos subtipos 2, 3 e 6 .

Contudo, foi encontrada uma predominância do sexo masculino $(78,6 \%)$ no subtipo DNM. Esse fato é frequentemente observado nos estudos de algumas DNM, sobretudo na esclerose lateral amiotrófica, onde há predominância do sexo masculino, na proporção geral de 1,5:1 em relação ao sexo feminino (STATLAND JM, et al., 2015).

Na presente série, quando as três variáveis (subtipo clínico, repetição do trinucleotídeo CAG e sexo do paciente) foram analisadas em único grupo, foi identificado que todas atuam conjuntamente, influenciando a idade de início do paciente. Assim, a maior precocidade dos sinais e sintomas da doença está associada a pacientes do sexo masculino com o subtipo 1 e que apresentam, concomitantemente, uma grande expansão CAG.

A antecipação e as instabilidades meióticas da expansão CAG são repetidamente documentadas na AEC (DONIS KC, et al., 2016; SOUZA GN, et al., 2016). Embora a antecipação de sinais e sintomas ter sido identificada em ambos os genitores no presente estudo, foi observada forte tendência da antecipação ser mais significativa quando o alelo mutado foi herdado paternalmente.

Apesar dos motivos para esse evento ainda não serem completamente compreendidos, é sabido que os alelos transmitidos paternalmente são mais instáveis do que os maternos, o que pode explicar parcialmente a antecipação (SOUZA GN, et al., 2016), embora o viés paterno não seja tão profundo como acontece na doença de Huntington (PAULSON H, 2012). Entretanto, Souza GN, et al. (2016) mostraram que não houve diferença nas antecipações causadas por alelos maternos e paternos.

Nossos resultados também sugerem que a antecipação é mais evidente em pacientes do sexo masculino. No entanto, na presente população, esse fato pode ser justificado pelo início da doença mais precoce em homens. Quando a antecipação da doença foi calculada para cada subtipo clínico, encontrou-se significativa antecipação nos subtipos 1 e 6 .

Nossos achados também sugerem que o percentual da prole afetada não sofre influência do sexo do genitor. Por outro lado, uma distorção de segregação favorecendo a transmissão do alelo expandido já havia sido proposta por Souza GN, et al. (2016). Tal fato não foi observado na presente série, uma vez que ambos os genitores transmitiram o alelo mutado em menos de $50 \%$ das vezes.

\section{CONSIDERAÇÕES FINAIS}

A população AEC3/DMJ aqui estudada apresenta algumas peculiaridades quando comparadas a pacientes com diferentes origens geográficas, sobretudo em relação às diferentes médias de idade de início masculina e feminina. As possíveis diferenças existentes entre os gêneros não foram elucidadas no presente estudo, mas permitiram levantar a hipótese de que genes modificadores fenotípicos possam modular a tendência para um início mais precoce ou tardio da doença na população estudada. Mais estudos são necessários para o reconhecimento das variáveis que influenciam o fenótipo e da forma com que interferem na população, a fim de se melhorar a qualidade de vida dos pacientes e de se aperfeiçoar o manejamento da doença.

\section{AGRADECIMENTOS E FINANCIAMENTO}

Agradecemos a todos os participantes do estudo por sua cooperação e apoio. Este trabalho foi financiado pelo Conselho Nacional de Desenvolvimento Científico e Tecnológico (CNPq) (4019942010-4 e Edital 01/2016 - Edição 2016), Coordenação de Aperfeiçoamento de Pessoal de Nível Superior (CAPES) (USP/RPMedicine (Neurology): 33002029012P3) e FAEPA, Brasil. 


\section{REFERÊNCIAS}

1. AKÇIMEN F, et al. Genome-wide association study identifies genetic factors that modify age at onset in MachadoJoseph disease. Aging, 2020, 12(6): 4742-4756

2. BETTENCOURT C, et al. The APOE epsilon2 allele increases the risk of earlier age at onset in Machado-Joseph disease. Arch Neurol, 2011; 68(12): 1580-1583.

3. BETTENCOURT C, LIMA M. Machado-Joseph Disease: from first descriptions to new perspectives. Orphanet J Rare Dis, $2011 ; 6(35):$ 1750-1172.

4. CHEN S, et al. Mitochondrial NADH Dehydrogenase Subunit 3 Polymorphism Associated with an Earlier Age at Onset in Male Machado-Joseph disease Patients. CNS Neurosci Ther, 2015; 22(1):38-42.

5. CHEN YS, et al. Identifying Therapeutic Targets for Spinocerebellar Ataxia Type 3/Machado-Joseph Disease through Integration of Pathological Biomarkers and Therapeutic Strategies. Int. J. Mol. Sci, 2020; 21: 3063.

6. CHEN Z, et al. (CAG)n loci as genetic modifiers of age-at-onset in patients with Machado-Joseph disease from mainland China. Brain, 2016; 139(8):41.

7. CINTRA VP, et al. Mutational screening of 320 Brazilian patients with autosomal dominant spinocerebellar ataxia. $J$ Neurol Sci, 2014; 347: 375-379.

8. DE CASTILHOS RM, et al. Spinocerebellar ataxias in Brazil--frequencies and modulating effects of related genes. Cerebellum, 2014; 13(1): 17-28.

9. DE MATTOS EP, et al. Age at onset prediction in spinocerebellar ataxia type 3 changes according to population of origin. Eur J Neurol, 2019; 26(1): 113-120.

10. DONIS KC, et al. Spinocerebellar ataxia type 3/Machado-Joseph disease starting before adolescence. Neurogenetics, 2016; 17(2): 107-113.

11. DU MONTCEL S, et al. Modulation of the age at onset in spinocerebellar ataxia by CAG tracts in various genes. Brain, 2014; 137(9): 2444-2455.

12. FARRAR MA, et al. Motor cortical dysfunction develops in spinocerebellar ataxia type 3. Clinical Neurophysiology, 2016; 127: 3418-3424.

13. FURTADO GV, et al. State biomarkers for Machado Joseph disease: Validation, feasibility and responsiveness to change. Genetics and Molecular Biology, 2019; 42(1): 238-251.

14. MARTINS S, et al. Modifiers of (CAG)n instability in Machado-Joseph disease (MJD/SCA3) transmissions: an association study with DNA replication, repair and recombination genes. Human Genetics, 2014; 133: 1311-1318.

15. MERGENER R, et al. Variation in DNA Repair System Gene as an Additional Modifier of Age at Onset in Spinocerebellar Ataxia Type 3/Machado-Joseph Disease. Neuromolecular Med, 2020; 22(1): 133-138.

16. MORO A, et al. Spinocerebellar ataxia type 3: subphenotypes in a cohort of Brazilian patients. Arq Neuropsiquiatr, 2014; 72(9): 659-662.

17. PAULSON H. Machado-Joseph disease/spinocerebellar ataxia type 3. Ataxic Disorders, 2012; 437-449.

18. SEIDEL K, et al. Brain pathology of spinocerebellar ataxias. Acta Neuropathol, 2012; 124: 1-21.

19. SEQUEIROS J, et al. Consensus and controversies in best practices for molecular genetic testing of spinocerebellar ataxias. Eur J Hum Genet, 2010; 18(11): 1188-1195.

20. SOUZA GN, et al. Spinocerebellar ataxia type 3/Machado-Joseph disease: segregation patterns and factors influencing instability of expanded CAG transmissions. Clin Genet, 2016; 90: 134-140.

21. STATLAND JM, et al. Patterns of Weakness, Classification of Motor Neuron Disease, and Clinical Diagnosis of Sporadic Amyotrophic Lateral Sclerosis. Neurol Clin, 2015; 33(4): 735-748.

22. TEIVE HG, et al. The Geographic Diversity of Spinocerebellar Ataxias (SCAs) in the Americas: A Systematic Review. Movement Disorders Clinical Practice 2019; 6(7): 531-540.

23. VALE J, et al. Autosomal dominant cerebellar ataxia: frequency analysis and clinical characterization of 45 families from Portugal. Eur J Neurol, 2010; 17(1):124-128.

24. WANG C, et al. Analysis of the GGGGCC Repeat Expansions of the C9orf72 Gene in SCA3/MJD Patients from China. PLoS One, 2015; 10(6): 1-9.

25. WYNER N, et al. Forensic Autosomal Short Tandem Repeats and Their Potential Association with Phenotype. Front Genet. 2020; 11: 884 .

26. ZHANG B, et al. Clinical manifestations and gene mutation in a case of Machado-Joseph disease. Neural Regen Res, 2012; 7(35): 2842-2847. 